\title{
Pharmacists for Patient Safety
}

\author{
Patricia Macgregor
}

$\mathrm{T}$

he expectation of the health care system is better care for patients, care that is standards-based and outcomes-driven. This expectation is founded on the dual principle that every patient deserves to be safe and every patient deserves optimal medication therapy. I have no doubt that every pharmacist believes this principle, as I also have no doubt that we try to put it into practice. Yet do we accomplish as much as possible to ensure that patients are safe in our hands and that they have the best care for their needs? We each have a role to play in ensuring safety and preventing harm, but do we embrace this role as we should?

New models of care give pharmacists the opportunity to be at the forefront of serving patients better and improving health care outcomes. Pharmacy leaders need to enhance awareness, both within and outside the profession, of the value that pharmacists bring as providers of patient care, not just as suppliers of medication, and pharmacists must demonstrate this value in their delivery of patient care.

How do we want to be known and measured as a profession: by the impact of medication safety incidents and system failures ... or by the improvements we make in patient safety and outcomes? Let's invest our efforts in driving optimal care for patients.

Pharmacists are not alone in their quest for patient safety and quality care. Health care organizations place significant focus on and demand accountability for these aspects of performance, as do professional colleges and safety organizations and networks, such as the Canadian Patient Safety Institute (CPSI), the Institute for Safe Medication Practices Canada, and Children's Hospitals' Solutions for Patient Safety. The directions of these and similar organizations and networks align well with those of the pharmacy profession and the Canadian Society of Hospital Pharmacists (CSHP). Under the key area of Pharmacists' Practice, CSHP's
2015-2018 Strategic Plan will include new partnering opportunities to improve knowledge translation and patient health outcomes. Among many envisioned partnerships, CSHP will engage with other stakeholders in CPSI's Integrated Patient Safety Action Plan to implement actions related to surgical care safety, medication safety, and infection prevention and control.

As the traditional roles of pharmacists and technicians change with incoming technology for medication dispensing, there are increasing opportunities for alternative roles in a collaborative clinical environment, including growing potential for advanced practice and specialization. Stewardship, in terms of both therapeutic decisions and advocacy, will be of increasing value. Pharmacists are also in a unique position to address the challenges inherent in the transfer of care between hospitals/ health care systems and the community, and have the chance to develop their roles in medication optimization and adherence, prevention of illness and safety incidents, adoption of best practices, patient self-management and monitoring, and collaborative health care. Together with other members of the health care team, we can make a difference.

Patricia Macgregor, BSc, RPh, MRPharmS, MHSc, CHE, is Past President and Vision Liaison for the Canadian Society of Hospital Pharmacists. 